\title{
Numerical Study of the Cavitating Flow through a Venturi Section by Means of OpenFOAM and Gmsh Tool
}

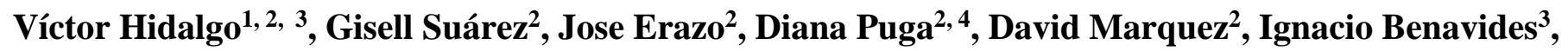 \\ Esteban Valencia ${ }^{1}$, XianWu Luo $^{4}$ \\ ${ }^{1}$ Escuela Politécnica Nacional, Departamento de Ingeniería Mecánica. \\ Av. Ladrón de Guevara E11-253, Quito 170517, Ecuador. \\ victor.hidalgo@epn.edu.ec \\ ${ }^{2}$ Escuela Politécnica Nacional, Laboratorio Mecánica-Informática, \\ Av. Ladrón de Guevara E11-253, Quito 170517, Ecuador. \\ ${ }^{3}$ Universidad Técnica del Norte, Ingeniería Automotriz, \\ Av. 17 de Julio 5-21, Ibarra 100105, Ecuador. \\ ${ }^{4}$ Tsinghua University, State Key Laboratory of Hydro Science \& Engineering \\ Beijing 100084, China.
}

\begin{abstract}
The present work focuses on the numerical study of the cavitating flow through a Venturi section by applying a scale adaptive simulation model. This study aims to understand the topology and unstable behavior of the cavitation cloud, which includes cavities formation as well as its collapse. Based on computational fluid dynamics, a methodology was developed to analyze the different stages of cavitating flow under the open-source guidelines. In this context, the numerical study was performed using OpenFOAM's environment with the help of the Gmsh meshing tool. For the resolution of Navier-Stokes equations, the PIMPLE algorithm was used considering a RANS equations approximation. Besides, the k-omega SST SAS turbulence model and the Zwart-Gerber-Belamri (ZGB) cavitation model were applied to study phase changes that occurs in the phenomenon. The obtained results of the present research show that the proposed methodology can reproduce the cavitation problem through a Venturi section with enough accuracy compared with experimental data and previous numerical simulations.
\end{abstract}

Keywords: Numerical study, cavitation, Venturi, Gmsh, OpenFOAM

\section{Introduction}

The phenomenon of cavitation has been a topic of great interest in the field of hydraulic engineering due to the negative consequences that it produces on turbomachines such as increase cost of repair and maintenance, reduction of efficiency, among others. For this reason, numerous studies have been carried out around the world with the purpose of understand the behavior of this phenomenon

Jian W. et al. [1] produced simulations of cavitating flow totally compressed to solve the formation of shock waves in the cloud collapse. In this study, ANSYS-Fluent was used with the k- $\varepsilon$ RNG turbulence model and the Zwart-Gerber-Belamri cavitating model. Two high-pressure peaks were found during the cavitation cycle, which were related to the detachment, and collapse that contributed to the gradual growth of erosion damage. It was concluded that the numerical models used in this study have great potential to the development of prediction techniques. On the other hand, Petrovsek M. et al. [2] made simultaneous observations of the cavitation structures and the surface of an aluminum sheet in a Venturi section. Two highspeed cameras showed the detachment sequence and cloud collapse with its corresponding damage due to erosion. It was concluded that the damaged occurred during the cavitation cloud collapse and the cloud size and its distance to the wall at the moment it collapses does not influence the damage extension. Furthermore, an irregular cloud causes greater damage to the aluminum sheet.

Similarly, Dural M. et al. [3] produced simultaneous observations of the cavitation structures in a Venturi section. A thin aluminum sheet was mounted because of its tendency to suffer severe damages due to cavitation under short periods. Moreover, high speed cameras registered the cavitation structures from several perspectives and the sheet surfaces. It was concluded that there are five damage mechanisms, being the most distinctive the collapse of a spherical cloud type 
"horseshoe" and "twister". Furthermore, wells were found during the detachment of the cavitation cloud and near the stagnation point closing of the adjoin cavity. For the hydro blade convex plane with a semi-circular obstacle, Escaler X. et al. [4] focused on the behavior of unstable cavitation around a convex hydroplane. The Zwart-Gerber-Belamri cavitation model was implemented on OpenFOAM based on the ILES model with a structured mesh by using Gmsh and Salome. Besides, numerical results were compared with hydrodynamic conditions produced experimentally in a highspeed cavitation tunnel. It was concluded that the mentioned numerical method can be used to investigate unstable cavitation around hydrofoils with satisfactory accuracy.

Hidalgo V. [5] developed a model for erosion due to cavitation based on the energy released during the collapse of the cavities and the assumption of homogeneous mixture flow. The model was developed through optimization of numerical simulation of the partially unstable cavitation, considering the asymmetric conditions of condensation and vaporization. The Zwart-Gerber-Belamri cavitation model with the ILES turbulence model were implemented in OpenFOAM. Simulations of the NACA0015 and NACA66 hydrofoils convex planes were performed using structured meshes produced in Gmsh and Salome. Results demonstrated the procedure of unstable cavitation, the cavity on the attack edge, the re-entering jet, the detachment and cavity collapse. The numerical results matched experimental data obtained by other sources.

In contrast, Li Z [6]. focuses on the behavior of unstable cavitation flow of hydrofoils. The NACA0015 and NACA0018-45 profiles were studied with structured mesh using RANS method implemented in Ansys FLUENT. It was discovered that the dynamic reduction of cavitation can only be obtained after the attenuation of Eddy viscosity on the region with higher vapor fraction. The study of fluid dynamics can be developed in three different ways, experimental, theoretical and numerical. The last one is related to CFD, which can predict the fluid flow through solving mathematical governing equations [7]. In this study, a discussion of advantages and disadvantages of CFD with respect to the experimental study and in the same way among the simulation software are presented. Besides, a general form of application of numerical studies is mentioned.

Previous numerical studies of unstable cavitation demonstrate that the principal aspects of this phenomenon can be reproduced accurately by using the free open software OpenFOAM. It captures precisely the formation of re-entering jet, generation and cavitation cloud collapse and the cavitating vortex in the flow. Based on those studies, the following research tries to reproduce the cavitation phenomenon throughout a numerical study, aiming to capture and analyse the formation of the cavity in the venturi section.

\section{Methodology}

This section details the methodology used for the mesh generation for a numerical study of the cavitating flow through a Venturi section, data processing and data analysis, and it is showed in Fig. 1. The first step was carried out the literature review of previous studies. Then, the geometric model is obtained to achieve the two-dimensional meshing of the Venturi by using Gmsh tool. Finally, this mesh is used to perform a numerical simulation of the phenomenon in OpenFOAM.

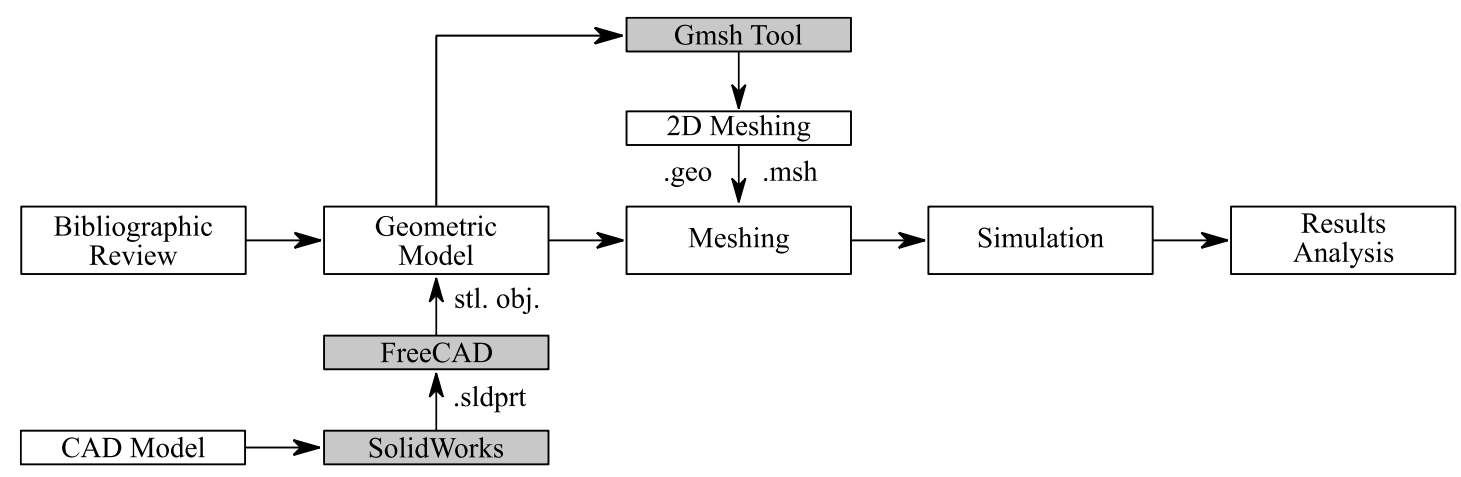

Fig. 1: General methodology. 


\subsection{Geometric Model}

The solid scheme is obtained using CAD programs. In the present work SolidWorks is used to export solids in .STL format. Then, FreeCAD is used for the generation of new objects (.obj, .stl) because SolidWorks cannot export files in these formats. These new objects allow the definition of parts, positioning with respect to the coordinate axes and help in the subsequent location of refinement zones. The Venturi section geometry dimensions were taken from Dular et. al [1], and it is depicted in Figure 2.

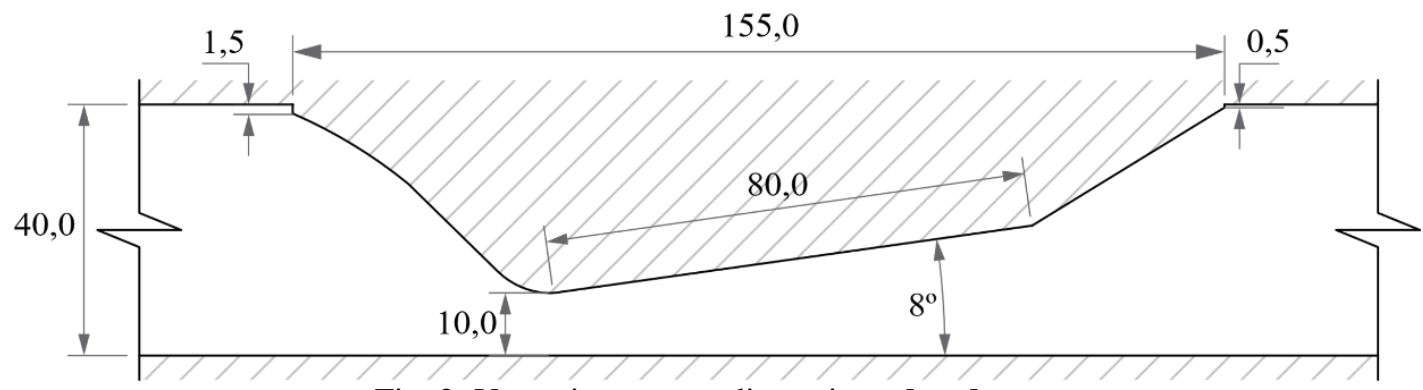

Fig. 2: Venturi geometry dimensions. [mm]

(Source: [1]).

\subsection{Meshing}

\section{Computational Domain:}

The Venturi section was located within the computational domain defined by the string length "c". The distance of the flow inlet and outlet was increased to two and four times the value of the string, respectively [8]. The value of the string is $0.15 \mathrm{~m}$ as shown in Fig. 3.

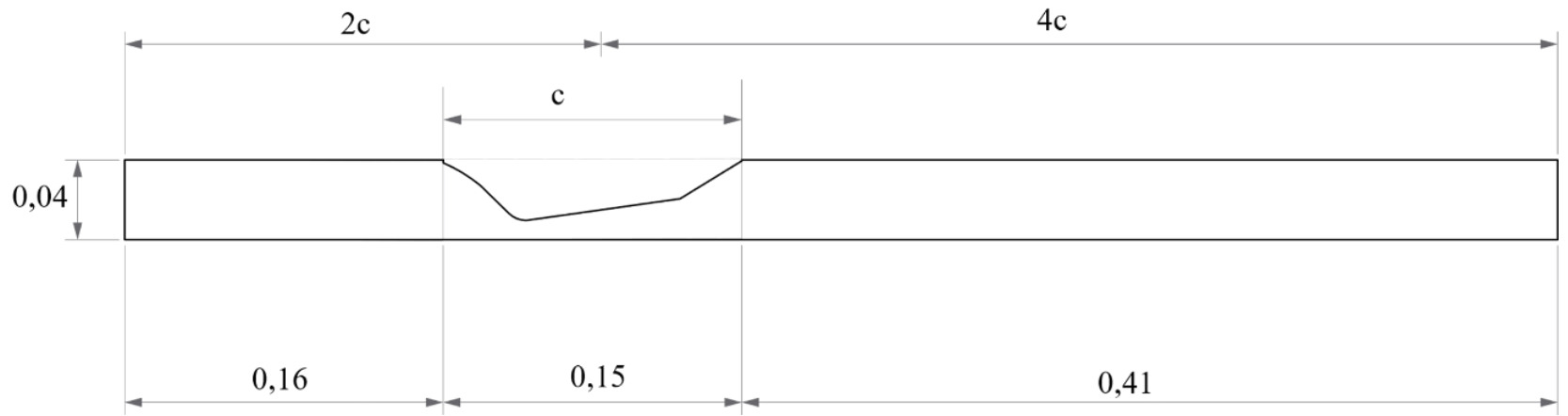

Fig. 3: Venturi geometry dimensions depending on the string length [m].

\section{Mesh Generation:}

Mesh is the discretization of the study domain, it means the division of the general domain into small volumes in which the algebraic equations are solved to analyse the behaviour of the phenomenon and the computational methods to solve differential equations [8]. The 2D mesh is obtained from the geometric model, and it needs characteristic points that allow the geometry definition Gmsh is used to obtain Venturi mesh. 


\section{Two-dimensional Meshing:}

The OpenFOAM software offers the opportunity to import meshes from other software by transforming the program through its own commands. For this reason, Gmsh software was used for mesh generation. The methodology for mesh generation is shown in Fig. 4.

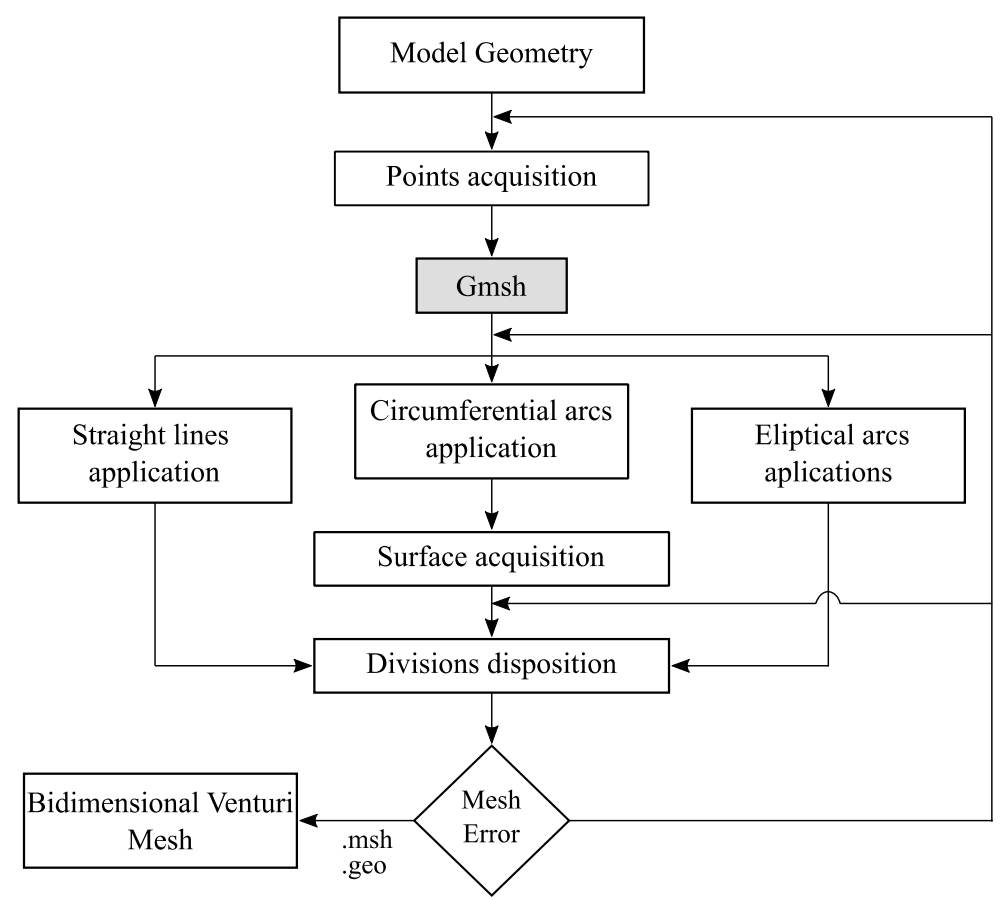

Fig. 4: Mesh generation methodology.

In order to generate a structured mesh throughout the domain, it was necessary to consider 27 points and 10 surfaces, as shown in Fig. 5a. The coordinates of each point were necessary to distribute them properly and locate the corresponding surfaces. To avoid a high slant and an abrupt orthogonality change, the lines (that must have the same properties and divisions) were identify as Fig. $5 \mathrm{~b}$ indicates.

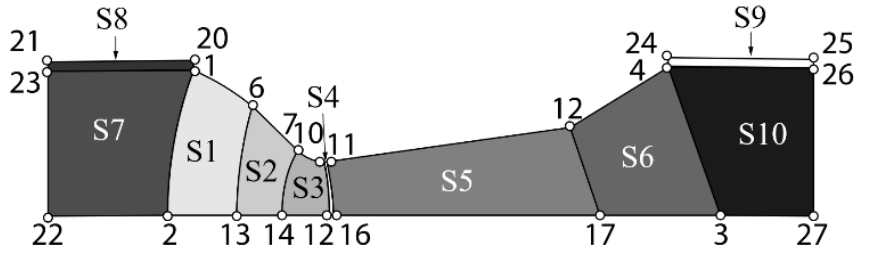

$5 \mathrm{a}$

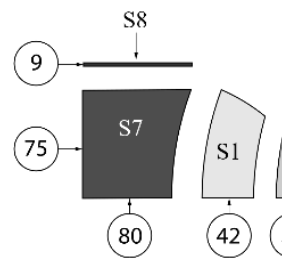

80

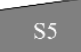

(200)

$5 b$

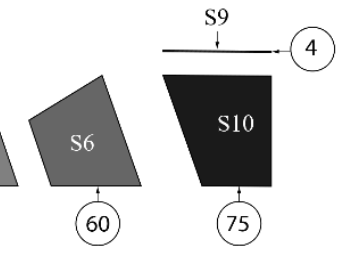

60

75

Fig. 5: Venturi Domain. 5a. Venturi domain points and sections. 5b. Division lines of each domain section.

\subsection{Equation}

The Zwart-Gerber-Belamri (ZGB) cavitation model is used in the present work and it is shown in equation 1, where Fv and $\mathrm{Fc}$ are the calibration constants for vaporization and condensation. On the other hand, $r_{n u c}$ is the nucleation site fraction, and $R_{B}$ is the typical bubble radius size in water $\dot{m}^{+}$, and $\dot{m}^{-}$are the mass flow rates for the two interphases $p$ is the local pressure, $p_{v}$ is the phase-change threshold pressure, $\rho_{v}$ and $\rho_{l}$ are the vapor and liquid density respectively $\alpha$ is the vapor 
fraction. Furthermore, the model was set using $\mathrm{Fv}=300$ and $\mathrm{r}_{\mathrm{nuc}}=5 \times 10-6$, which are based on Morgut et al., and $\mathrm{Fc}$ was used the model default value defined as $\mathrm{Fc}=0.01$.

$$
\dot{m}=\left\{\begin{array}{ll}
\dot{m}^{+}=F_{v} \frac{3 r_{n u c}(1-\alpha) \rho_{v}}{R_{B}} \sqrt{\frac{2}{3}\left(\frac{p_{v}-p}{\rho_{l}}\right)}, & \text { for } p<p_{v} \\
\dot{m}^{-}=F_{c} \frac{3 \alpha \rho_{v}}{R_{B}} \sqrt{\frac{2}{3}\left(\frac{p_{v}-p}{\rho_{l}}\right)}, & \text { for } p>p_{v}
\end{array} .\right.
$$

\section{Results and Discussion}

In order to obtain good mesh quality and avoid induction of results, further refinement is performed in the interest area. A mesh independence study was conducted over four grid resolutions with different densities starting from coarse to refined. Furthermore, a velocity and pressure analysis were performed over 10 localised points over the free flow along the Venturi geometry as shown in Fig. 6.

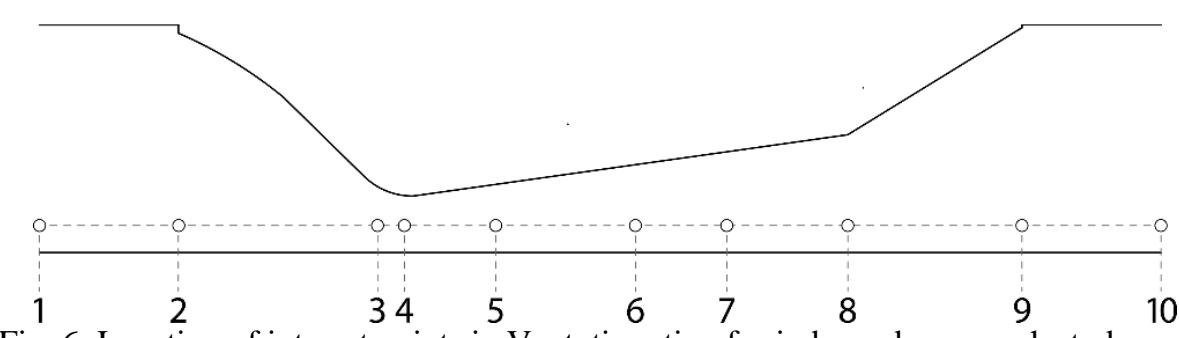

Fig. 6: Location of interest points in Ventuti section for independence mesh study.

The mesh independence study was carried out using Gmsh and over a stabilization time of $t=1,500 e^{-2}[s]$. Table 1 shows the free flow velocity whereas Table 2 . shows the pressure analysis at the different points. Both Tables demonstrate that Mesh 2 (M2), which has 41.036 elements, shows mesh independence. The velocity and pressure data changes negligibly with increasing number of elements from M2 to M3 and M4.

Table 1: Mesh independence study considering four meshes - Velocity $[\mathrm{m} / \mathrm{s}]$.

\begin{tabular}{|c|c|c|c|c|c|}
\hline \multicolumn{2}{|c|}{$\mathbf{N}^{\circ}$ Elements } & 18.845 & 41.036 & 56.770 & 61.949 \\
\hline Points & Distance $(\mathbf{x})$ & M1 & M2 & M3 & M4 \\
\hline 1 & 0,010 & 6,175 & 6,175 & 6,175 & 6,175 \\
\hline 2 & 0,248 & 8,164 & 7,671 & 7,647 & 7,653 \\
\hline 3 & 0,285 & 22,992 & 23,807 & 23,807 & 23,802 \\
\hline 4 & 0,290 & 28,023 & 28,029 & 28,031 & 28,029 \\
\hline 5 & 0,307 & 31,990 & 31,019 & 31,006 & 31,005 \\
\hline 6 & 0,333 & 31,145 & 31,088 & 31,077 & 31,081 \\
\hline 7 & 0,350 & 31,138 & 31,094 & 31,083 & 31,090 \\
\hline 8 & 0,370 & 31,016 & 31,104 & 31,033 & 31,089 \\
\hline 9 & 0,405 & 31,114 & 30,851 & 30,836 & 31,174 \\
\hline 10 & 0,820 & 11,164 & 11,142 & 11,155 & 11,146 \\
\hline
\end{tabular}


Table 2: Mesh independence study considering four meshes - Pressure $\mathrm{P}[\mathrm{Pa}]$

\begin{tabular}{|c|c|c|c|c|c|}
\hline \multicolumn{2}{|c|}{$\mathbf{N}^{\circ}$ Elements } & $\mathbf{1 8 . 8 4 5}$ & $\mathbf{4 1 . 0 3 6}$ & $\mathbf{5 6 . 7 7 0}$ & $\mathbf{6 1 . 9 4 9}$ \\
\hline Points & Distance (x) & M1 & M2 & M3 & M4 \\
\hline 1 & 0,010 & 467.232 & 464.862 & 464.438 & 464.411 \\
\hline 2 & 0,248 & 456.596 & 454.330 & 453.912 & 453.870 \\
\hline 3 & 0,285 & 203.735 & 201.423 & 201.085 & 201.043 \\
\hline 4 & 0,290 & 94.704 & 92.363 & 91.991 & 91.974 \\
\hline 5 & 0,307 & 4.694 & 4.454 & 4.445 & 4.443 \\
\hline 6 & 0,333 & 2.400 & 2.371 & 2.366 & 2.365 \\
\hline 7 & 0,350 & 2.386 & 2.359 & 2.361 & 2.356 \\
\hline 8 & 0,370 & 5.666 & 2.397 & 3.563 & 2.383 \\
\hline 9 & 0,405 & 2.404 & 4.818 & 4.372 & 4.866 \\
\hline 10 & 0,820 & 2.421 & 2.395 & 2.351 & 2.212 \\
\hline
\end{tabular}

Consequently, using M2 a horizontal progression parameters variation of each surface is accomplished in upper and lower edges of the geometry to achieve a smooth transition. The mesh is disposed in the "x-z" direction, and the "y" direction has a negligible thickness (two dimensions analysis). The 3.0.6 version of Gmsh software was used.

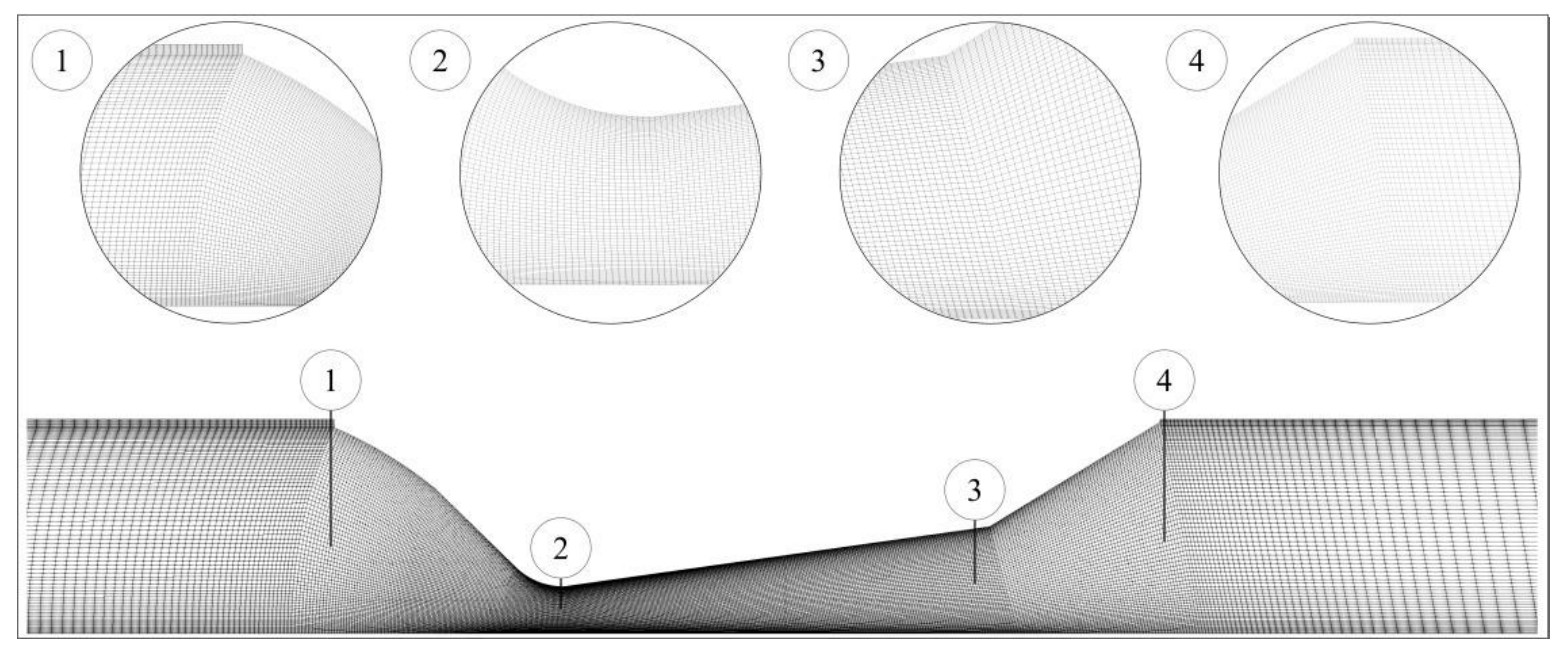

Fig. 7: Domain meshing.

The M2 mesh with 41036 elements showed an instability between iterations 250 and 2000 at the 0,500 e- 3 to 3,000 e-3 time interval as shown in Fig. 8. Within this time period, the cavitation cycle occurs. After $\mathrm{t}=3,5$ e-3; generalized cavitation was observed, which implies similarity between the results obtained in this study and previous ones. 


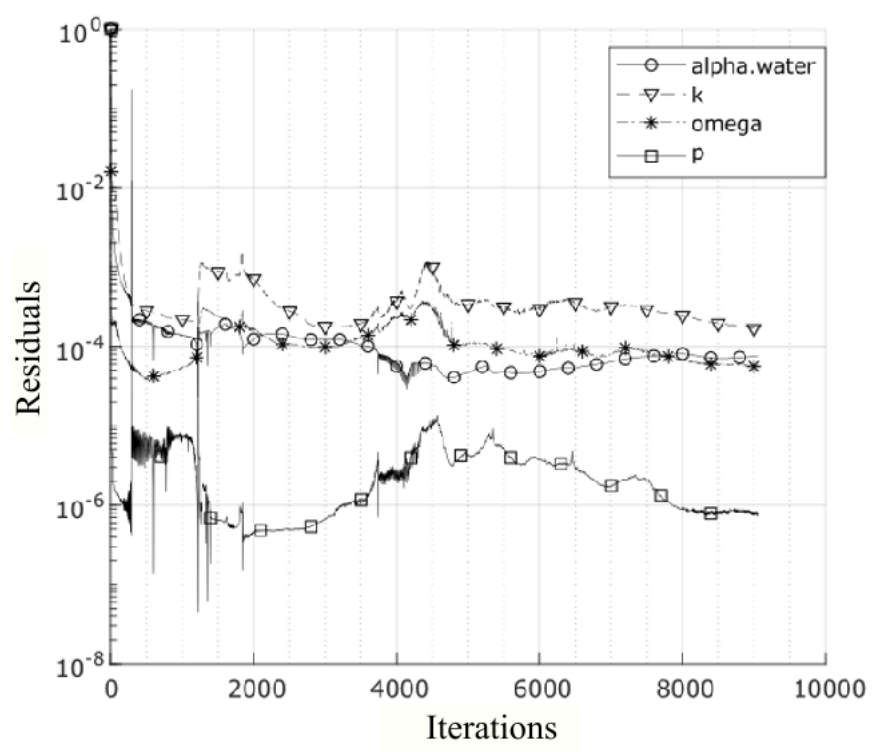

Fig. 8: Residuals vs. Iterations.

The numeric simulation was validated with Dular et al. experimental and computational previous studies. The cavitation cycle matches with the one presented in Dular et al work, but the detachment and collapse time were different. The time interval used for this work was $\Delta \mathrm{t}=9000 \mathrm{e}-6$ and Dular's work one was $\Delta \mathrm{t}=1600 \mathrm{e}-4$. The detachment time obtained was at $\mathrm{t}=$ to $+80 \Delta \mathrm{t}$ and the collapse at $\mathrm{t}=\mathrm{to}+175 \Delta \mathrm{t}$ and Dular et al results showed the detachment at $\mathrm{t}=$ to $+20 \Delta \mathrm{t}$ and collapse at $\mathrm{t}=$ to $+45 \Delta \mathrm{t}$. Furthermore, peak pressure sections in the Venturi matched the ones presented by Dular et al. obtaining a pressure during the cloud collapse of 3 e $7[\mathrm{~Pa}]$ and a lower 4,8 e5 [Pa] for the detachment.

\section{Conclusion}

The obtained results of the present research show that the proposed methodology can reproduce the cavitation problem through a Venturi section with enough accuracy compared with experimental data and previous numerical simulations. From this study, it can be concluded that the M2 mesh produces values within acceptable range for $y+$, Courant Number and Omega number that allows capturing the cavitation cycle in a Venturi tube in time intervals of 9e-6. Moreover, the use of the turbulence k-omega SST SAS model with the ZGB cavitation model predicts precisely the asymmetric cloud collapse, however it presents difficulties when interpreting correctly the detachment.

\section{Acknowledgements}

The authors gratefully acknowledge the support provided by Computer Laboratory of Escuela Politécnica Nacional for the development of this study, which is part of the research projects PIJ 17-13 and PII-DIM-2019-06.

\section{References}

[1] W. Jian, M. Petkovšek, L. Houlin, B. Širok, and M. Dular, "Combined numerical and experimental investigation of the cavitation erosion process," J. Fluids Eng. Trans. ASME, vol. 137, no. 5, May 2015.

[2] M. Petkovšek and M. Dular, "Simultaneous observation of cavitation structures and cavitation erosion," Wear, vol. 300, no. 1-2, pp. 55-64, Mar. 2013.

[3] M. Dular and M. Petkovšek, "On the mechanisms of cavitation erosion - Coupling high speed videos to damage patterns,” Exp. Therm. Fluid Sci., vol. 68, pp. 359-370, Nov. 2015.

[4] X. Escaler, E. Egusquiza, M. Farhat, F. Avellan, and M. Coussirat, "Detection of cavitation in hydraulic turbines," Mech. Syst. Signal Process, vol. 20, no. 4, pp. 983-1007, May 2006. 
[5] V. Hidalgo, "Numerical study on unsteady cavitating flow and erosion based on homogeneous mixture assumption," 2016.

[6] Z. R. Li, M. Pourquie, and T. Van Terwisga, "Assessment of cavitation erosion with a URANS method," J. Fluids Eng. Trans. ASME, vol. 136, no. 4, Apr. 2014.

[7] A. Bakker and E. Marden Marshall, "Computational Fluid Dynamics (Pre-Print)," Encyclopedia of Chemical Processing. 2016.

[8] C. Poveda and S. Simbaña, "Estudio numérico y experimental de caudales óptimos para secado de grano de cacao.," 2018. 